health organisations, and professional societies, focusing on vaccination policies and practices in occupational settings.

Result Many vaccines are available for workers exposed to certain biological agents, while others are imminent. However, systematic, representative data on occupational immunisation policies, practices and coverage are lacking. Most pertain to HCWs, Influenza and HBV and coverage is sub-optimal. Variations exist by country and region for legislation, policies, schedules, groups requiring vaccination, implementation. Relevant issues include: access to vaccines; cost-effectiveness; immunisation coverage; identification susceptible of workers at-risk; fitness for work; involvement of all stakeholders.

Discussion Immunisation is an additional preventive tool to conventional workplace interventions, very effective for biohazard containment. A specific immunisation policy and practice is a strategic priority for individual workers, different workforces and the public health system. The main Occupational Health Professionals' (OHP) responsibilities are: individual, activity and area risk assessment; evaluation and provision of cost-effective vaccines; obtaining consent (or refusal) following counselling; managing non-responders and those who refuse vaccinations; career guidance; remaining updated. Mandatory immunisation should be explored in selected circumstances. OHP, employers and workers share the responsibility to implement the WHO Global Vaccine Action Plan to expand access to immunisation to all susceptible workers and strengthen routine immunisation. Policies and services should reside in the realm of Corporate Social Responsibility and be harmonised across industries, regions and countries with the ultimate goal to control and eradicate VPD in workplaces.

\section{3d OCCUPATIONAL INFECTIOUS AGENTS (OIA) IN HOSPITAL FACILITIES - INTERFACE BETWEEN OCCUPATIONAL AND PUBLIC HEALTH}

K Reijula. Professor of Occupational Health, Helsinki University, Helsinki, Finland

\subsection{6/oemed-2018-ICOHabstracts.593}

Introduction Mainly due to epidemic infections such as SARS and avian influenza, exposure prevention to occupational infectious agents (OIA) has become of increasing interest in hospitals. New models of action between different professionals in hospitals and improving the competence of hospital workers are urgently needed.

Methods Attitudes, knowledge and skills of hospital workers, action models in preventing OIA exposure and the performance of isolation rooms in preventing the spread of microbes in patient wards were assessed in Finnish hospitals. Multi-professional team work between personnel in hospitals and health care (both occupational and public health) but also with the management of hospital facilities were evaluated.

Result Best results in preventing exposure to OIA were found in hospitals where the competence of personnel was high and the staff was committed to use the best practices of exposure prevention. The quality in the performance of isolation rooms played a significant role in results. Close collaboration between occupational health and the staff of infectious diseases but also with public health was found necessary in improving the processes of exposure prevention.

Discussion Multi-professional collaboration between occupational health, the personnel of infectious diseases and public health seems to have a significant role in preventing exposure to OIA. Health care professionals need also to work together with the management of facilities to improve the quality of indoor environment and the performance of isolation rooms. The attitudes and competence of hospital staff in association with good performance of isolation rooms in hospitals are critical related to occupational safety and health of hospital workers.

\section{3e INNOVATIONS IN INDUSTRIAL HYGIENE APPROACHES TO INFECTION CONTROL}

TP Fuller. Illinois State University, Normal, Illinois, USA

\subsection{6/oemed-2018-ICOHabstracts.594}

Introduction Healthcare Acquired Infections (HAIs) continue to pose a major burden on healthcare facilities around the world with major human and financial consequences. Furthermore, infectious diseases acquired while on-the-job continue to be a significant cause of morbidity and fatality of healthcare workers. It is difficult to deny that more needs to be done to control hazardous infectious agents and protect patients and workers from exposure.

Methods Industrial hygiene approaches to environmental and workplace hazardous infectious agents including anticipation, recognition, evaluation, and control were used as the foundation to re-evaluate infectious agents in the healthcare environment. The latest advances in the industrial hygiene hierarchy of controls were investigated including engineering, administrative measures, and the use of Personal Protective Equipment.

Result The field of industrial hygiene has been expanding at a rapid pace to support the needs of infection control in healthcare. New technologies are being demonstrated and implemented on several fronts. The ability of hospitals to protect patients by reducing HAIs can be influenced by the ability to effectively utilise the new industrial hygiene approaches to infection control. In addition to protecting patients, the likelihood of worker infections will also be reduced.

Discussion Industrial hygiene approaches to infection control in healthcare are sometimes misunderstood and often underutilised. The advantages of integrating new technologies in identifying, and then destroying infections agents in the hospital environment, demonstrates significant potential for protecting both patients and workers from communicable diseases acquired in the healthcare system.

\section{HOSPITAL LENGTH OF STAY AND ASSOCIATED FACTORS AMONG CONFIRMED INFLUENZA PATIENTS ADMITTED IN KING ABDULAZIZ MEDICAL CITY, WESTERN SAUDI ARABIA}

${ }^{1}$ Fayssal Farahat*, ${ }^{1}$ Mohammed Abd Al Aziz* ${ }^{1}$ Omaima Hassan*, ${ }^{1}$ Asim AlSaedi ${ }^{*}$, ${ }^{2}$ Majid AlShamrani*. 'Infection Prevention and Control, King AbdulAziz Medical City, Jeddah; ${ }^{2}$ Infection Prevention and Control Program, Ministry of National Guard

\subsection{6/oemed-2018-ICOHabstracts.595}

Introduction Influenza can cause mild to severe illnesses and serious outcomes can result in hospitalisation or death. Clinical presentation and management of influenza cases in Saudi Arabia have been tackled especially during 2009 H1N1 pandemic, however, available data on influenza related hospital stay and determining factors in the country is limited. This study aims to assess factors that determine the length of stay 
(LOS) among hospitalised influenza confirmed cases in King Abdulaziz Medical City - western Saudi Arabia.

Methods A retrospective review of laboratory-confirmed influenza cases admitted to King Abdulaziz Medical City during the period from January to December 2016 was conducted. Identified variables included age, gender, influenza strains and associated comorbidities.

Results Among the 556 laboratory-confirmed influenza cases during the study period, 157 patients were admitted (28.2\%). More than half $(52.2 \%)$ of the admitted patients were females, $20.4 \%$ were $\leq 5$ years old, $51.6 \%$ were $>5-65$ years old and $28.0 \%$ were $>65$ years old. Healthcare workers represented $3.2 \%$ of the admitted patients. Influenza A-non H1N1 represented $65.6 \%$ followed by influenza B (36.3\%) and influenza A-H1N1 (10.8\%). Some patients have more than one episode of influenza. Most patients (65.0\%) were admitted for more than 2 days $(41.4 \%$ for $2-5$ days and $23.6 \%$ for more than 5 days). Length of stay was significantly longer among elderly patients ( $>65$ years old) and patients with DM, cardiac, chronic respiratory, immune deficiency diseases and with increased number of co-morbidities, however, no statistical significant association was reported according to influenza strains.

Conclusion Significant number of patients were admitted because of influenza for duration more than 2 days. Length of stay was associated with chronic morbidities and old age. Enhancement of immunisation programs especially for high risk patients is essential for reducing burden of influenza on patients and healthcare system.

\section{THE INCIDENCE OF COMMUNITY ACQUIRED PNEUMONIA BY OCCUPATION}

${ }^{1}$ Anil Adisesh*, ${ }^{1}$ Anne Theriault, ${ }^{2}$ Donna Mackinnon-Cameron ${ }^{2}$ Ardith Ambrose, ${ }^{3}$ Duncan Webster, ${ }^{3}$ Stefanie Materniak, ${ }^{4}$ Sylvie Trottier, ${ }^{4}$ Guy Boivin, ${ }^{4}$ Isabelle Chabot, ${ }^{5}$ Anne McCarthy, ${ }^{5}$ Stephanie Carson, ${ }^{5}$ Wanda Allen Shelly McNeil ${ }^{2} .{ }^{1}$ Dalhousie Medicine New Brunswick, Dalhousie University, Saint John, New Brunswick, Canada; ${ }^{2}$ Canadian Centre for Vaccinology, IWK Health Centre and Nova Scotia Health Authority, Dalhousie University, Halifax, Nova Scotia, Canada; ${ }^{3}$ Horizon Health, Saint John, New Brunswick, Canada; ${ }^{4}$ Centre Hospitalier Universitaire de Québec, Québec, Québec, Canada; ${ }^{5}$ The Ottawa Hospital, Ottawa, Ontario, Canada

\subsection{6/oemed-2018-ICOHabstracts.596}

Introduction Reversible, modifiable risk factors are associated with a greater risk of developing community acquired pneumonia (CAP). Welders of working age are 3.5 times more likely to die from pneumococcal pneumonia than workers in other jobs. A higher risk of CAP is seen in workers exposed to any type of metal fume and this excess risk is limited to below the age of 65 years, indicating a reversible susceptibility. Other causes of CAP may also be related to occupation or recent working conditions.

Methods At 12 sites across Canada patients admitted to hospital with CAP have been recruited to participate in a wider study. As a pilot we added questions regarding occupation. The information was coded using the Canadian National Occupational Classification (NOC) 2011. Data were used to calculate percentages and compare occurrences of pneumonia across occupations.
Results We obtained occupation data on 171 cases (now 671). The NOC codes were aggregated to the ten single digit NOC codes. Those in 'trades, and related fields' comprised $26 \%$ $(n=44)$ of cases when including retired workers. There was a significantly greater proportion of cases $32 \%(n=29, p=0.05$, $\mathrm{Chi}=3.834$ ) amongst current workers in 'trades and related occupations' compared to workers in all other jobs $68 \%$ $(n=62)$. There were five single digit NOC codes including 'trades and related occupations' where the proportion of cases amongst current workers was higher than in those retired.

Conclusion Our data suggests workers in 'trades and related fields' are more at risk of CAP with the proportion affected exceeding that of those employed in this group, 25.5\% (StatCan, 2011). Few studies have analysed occupations and exposure as risk factors for developing CAP. The reduction of cases in those retired 'trades and related occupations' compared to current workers may represent an occupational effect.

\section{LATENT TUBERCULOSIS INFECTION AMONG HEALTHCARE WORKERS AT A GENERAL HOSPITAL}

D Melo*, T Pinto, E Silva, M Bastos, L Pires, AP Sardo, F Mautempo. Occupational Health and Work Medicine Service, Centro Hospitalar do Baixo Vouga, Aveiro, Portugal

\subsection{6/oemed-2018-ICOHabstracts.597}

Introduction Healthcare workers (HCWs) have a higher risks of contracting tuberculosis $(\mathrm{TB})$ than general population. International and national policies recommend routine screening of latent tuberculosis infection (LTBI) as an essential component in the control and prevention of TB in healthcare facilities.

Methods From January 2008 to December 2016, 1759 hospital employees were screened for LTBI. Symptom assessment and chest X-ray were conducted to exclude active TB, and tuberculin skin test (TST) and/or QuantiFERON-TB Gold test (QFT) were performed to diagnose LTBI.

Results At the end of 2016, 1054 active workers were screened one or more times, totalizing 1810 screenings; $81.5 \%$ were female and $18.5 \%$ were male; mean age was 42 years. None were found to have active TB. LTBI prevalence in the screened population was $17.7 \%(n=187)$ : 101 individuals had a QTF positive test and 86 didn't perform QTF test but had a TST $\geq 15 \mathrm{~mm}$. The majority were positive for LTBI at the first screening $(n=110 ; 58.8 \%)$. Among the screened HCWs, medical aid assistants had the highest prevalence of LTBI (21.7\%), followed by nurses (19.4\%), administrative and supportive staff $(14.4 \%)$, while physicians had the lowest prevalence $(12.4 \%)$ of LTBI. QFT was negative in $47.3 \%$ of the individuals with TST $\geq 15 \mathrm{~mm} \quad(\mathrm{n}=61$, which 41 submitted to repetitive TST testing), and in $76.5 \%$ of the cases with TST $\geq 10 \mathrm{~mm}$ but $<15 \mathrm{~mm}$ ( $\mathrm{n}=65$, which 37 submitted to repetitive TST testing).

Discussion Since 2015, Portugal has been a low-incidence country regarding TB. The prevalence of LTBI in HCWs is relatively high as far as $17,7 \%$. As a result, active screening for TB and LTBI is needed for these workers. Screening with TST and QTF is a costeffective approach as high numbers of discordant TST positive/ QTF negative results are probably caused by BCG vaccination or boosting due to repetitive TST testing. 